\title{
Characterization of Cross-linking Structures in UV-cured Acrylic Ester Resin by MALDI-MS Combined with Supercritical Methanolysis
}

\author{
Hideki Matsubara, ${ }^{* 1}$ Shun-ichiro Hata, ${ }^{2}$ Yosuke Kondo, *2 Yasuyuki IshIDA,*3 \\ Hiroshi TAKIGAWA, ${ }^{* 4}$ and Hajime OHTANI*5† \\ *1 Aichi Industrial Technology Institute, Kariya 448-0003, Japan \\ *2 Department of Materials Applied Chemistry, Graduate School of Engineering, Nagoya University, \\ Nagoya 464-8603, Japan \\ *3 EcoTopia Science Institute, Nagoya University, Nagoya 464-8603, Japan \\ *4 Sumitomo Chemical Co., Ltd., Tsukuba 300-3266, Japan \\ *5 Department of Materials Science and Engineering, Graduate School of Engineering, \\ Nagoya Institute of Technology, Nagoya 466-8555, Japan
}

\begin{abstract}
The cross-linking structure of the ultra violet (UV)-cured resin prepared from dipentaerithritol hexacrylate (DPHA) was characterized by matrix-assisted laser desorption/ionization mass spectrometry (MALDI-MS) combined with supercritical methanolysis. The MALDI-mass spectrum of the decomposition products obtained by supercritical methanolysis contained a series of peaks of sodium-cationized methyl acrylate (MA) oligomers up to around $\mathrm{m} / \mathrm{z}=4000$ formed through selective cleavage and methylation occurred at ester linkages in UV-cured DPHA. Furthermore, in order to observe widely distributed sequence lengths in the cross-linking junctions, the decomposed products of the cured resin were then fractionated using size exclusion chromatography followed by the MALDI-MS measurements of the individual fractions. The MALDI-mass spectra of the lower molar mass fractions mainly consisted of a series of peaks of MA oligomers around $\mathrm{m} / \mathrm{z}$ values of several thousands, whereas those of higher molecular weight showed a broad peak up to $\mathrm{m} / \mathrm{z}$ ca. 180000. The observed distributions of the supercritical methanolysis products suggested that the network junctions in the given UV-cured resin were composed of up to around 2000 acrylate units.
\end{abstract}

(Received July 7, 2006; Accepted August 9, 2006; Published November 10, 2006)

\section{Introduction}

Ultraviolet (UV) curable acrylic esters are applied to many industrial products such as printing inks, coatings for optical fibers and disks, adhesives, dental materials, photoresist, and three-dimensional stereolithography. ${ }^{1-5}$ In order to utilize these in advanced technology, it is often requested to elucidate the cross-linking network structure of the resulting cured resins by UV-irradiation. However, it is not an easy task to analyze cured resins even using advanced spectroscopic methods mainly because of their insoluble nature. Recently, the authors characterized the chain length distribution of network junctions in UV-cured poly(ethylene glycol) diacrylate (PEDA) using pyrolysis-gas chromatography in the presence of tetramethylammonium hydroxide. ${ }^{6}$ In this case, methyl acrylate (MA) oligomers formed through thermally assisted hydrolysis and methylation selectively occurred at ester linkages in UVcured PEDA were attributed to the sequences of the network junctions in the cross-linking structure. The chain length distribution of the network junctions composed of up to six acryloyl units in the UV-cured PEDA was estimated from the peak intensities of the corresponding MA oligomers observed in

$\dagger$ To whom correspondence should be addressed.

E-mail: ohtani.hajime@ nitech.ac.jp the pyrogram. However, the network structure containing longer sequences comprised of more than seven acryloyl units was not elucidated because the corresponding higher MA oligomers were not observed in the pyrogram, mainly due to their lower volatility.

Meanwhile, Burkoth et al. evaluated the kinetic chain length of the network junctions of UV-cured dimethacrylated sebacic anhydride through matrix-assisted laser desorption/ionizationmass spectrometric (MALDI-MS) measurements of its decomposition products. ${ }^{7}$ This polymer was readily hydrolyzed in phosphate buffered saline at $\mathrm{pH} 7.4$ and $37^{\circ} \mathrm{C}$ during several hours into sebacic acid and poly(methacrylic acid). They observed that poly(methacrylic acid)s reflected the sequences of network junctions in the range between pentamer and 24-mer in the MALDI mass spectra. This approach, however, was limited for the loosely cross-linking polymer composed of the hydrophilic monomers and oligomers suitable for biodegradable applications. Such materials should be much easier to be hydrolyzed than practical UV-cured resins in the industrial fields with rigidly cross-linking structure essential for high chemical resistance and heat resistance.

On the other hand, supercritical fluids such as supercritical methanol have been utilized to depolymerize intractable polymers such as poly(ethylene terephthalate) $(\mathrm{PET})^{8}$ and phenol resin ${ }^{9}$ mainly for the purpose of plastic recycling. In the former instance, ${ }^{8}$ PET was almost completely decomposed in 


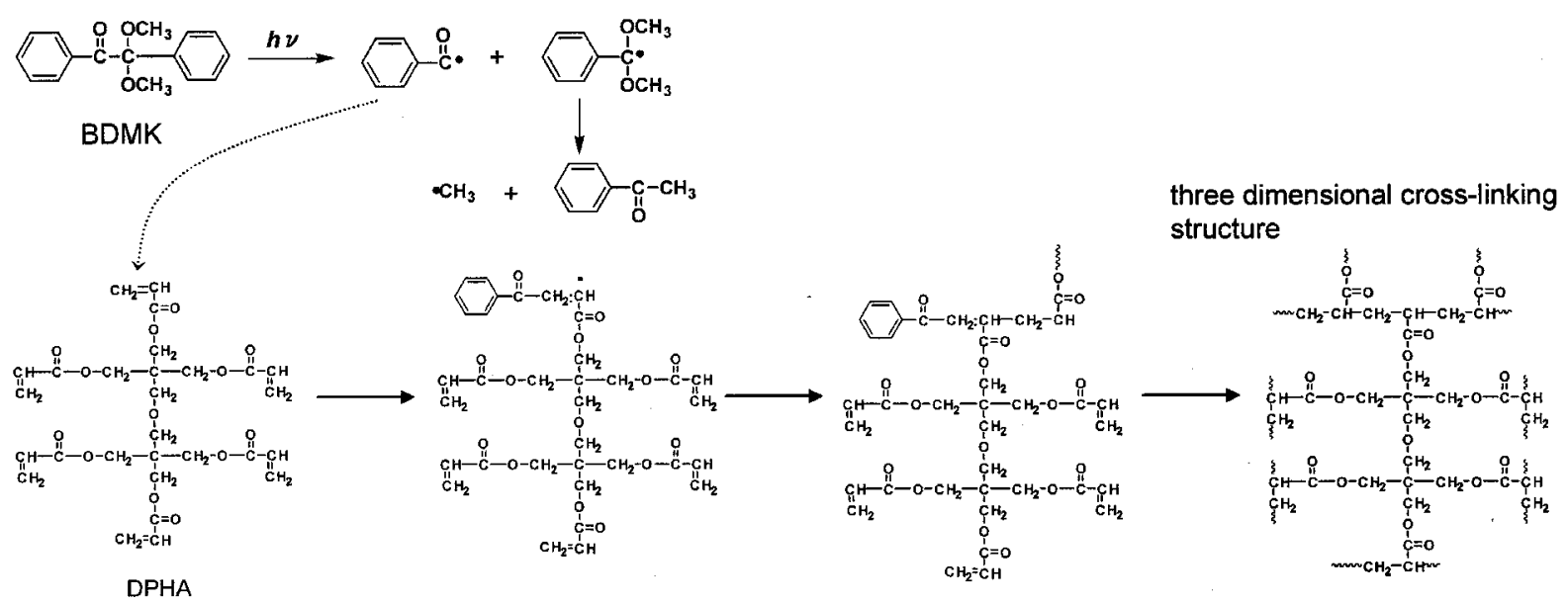

Fig. 1 Typical formation pathway of UV-cured DPHA.

methanol in 30 min under the supercritical conditions at $300^{\circ} \mathrm{C}$ and various pressures over 8.1 MPa. In this case, the ester linkages clove to give dimethyl terephthalate and ethylene glycol, although oligomeric products such as 4methoxycarbonyl(2-hydroxyethyl)benzoate were also produced with the yield of $40-50 \%$. This result suggests that the supercritical methanol is a potential solvent to cause selective cleavages of ester linkages with high efficiency even in the intractable polymeric materials.

In this work, MALDI-MS combined with supercritical methanolysis was used as an analytical method to characterize network structure of the UV-cured acrylic ester resin. First, UV-cured resins prepared from dipentaerythritol hexacrylate utilized in the industrial fields were subjected to supercritical methanolysis to achieve selective decomposition at the ester linkages. The products thus obtained were measured by MALDI-MS to observe the sequence distributions of the network junctions. Moreover, size exclusion chromatographic fractionation of the products was combined with MALDI-MS ${ }^{10}$ to evaluate appropriately the widely distributed sequences of network junctions.

\section{Experimental}

\section{Samples}

Dipentaerythritol hexacrylate (DPHA) was obtained from Kyoeisha Chemical. The photoinitiator, 2,2-dimethoxy-2phenylacetophenone (benzyl dimethyl ketal; BDMK, Wako Pure Chemical Industries) was used without further purification. A typical formation pathway of the UV-cured DPHA using BDMK as a photoinitiator is shown in Fig. 1. By absorbing UV light, BDMK decomposes through $\alpha$-splitting to give a benzoyl radical and a dimethoxybenzyl radical. The latter radical further decomposes into a methyl benzoate and a methyl radical. Each of these radicals should contribute to initiate photopolymerization although, only the initiation by a benzoyl radical was represented in Fig. $1 .^{11}$

The procedure for preparing UV-cured resin samples is basically the same as that described in our previous papers., ${ }^{6,12}$ Prepolymers were compounded by dissolving BDMK into DPHA at $70^{\circ} \mathrm{C}$ together with a small amount of 1,4benzoquinone (BQ; Tokyo Kasei Kogyo) (DPHA:BDMK:BQ = 100:3:0.5 in wt.). Here, BQ was added to prevent excessive chain growth of the cross-linking sequences. Then, each

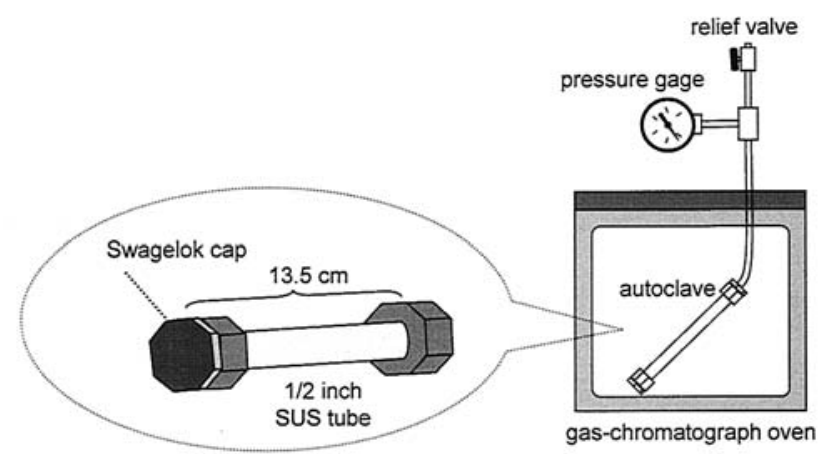

Fig. 2 Schematic diagram of degradation apparatus used in this work.

viscous prepolymer solution was poured into a laboratory dish to form a wet film with 500-600 $\mu \mathrm{m}$ thickness. UV-curing was carried out in air by passing the UV-curable prepolymer in the dish through the UV-curing equipment consisting of a conveyor, a medium-pressure mercury lamp $(80 \mathrm{~W} / \mathrm{cm}$ and total output $2 \mathrm{~kW}$ ) mounted in a dichroic mirror, and an infrared cutoff filter. The dosage for preparing UV-cured DPHA was determined to be $64 \mathrm{~mJ} / \mathrm{cm}^{2}$ by a UV-integrator (EYE Graphics UVPF-36, wavelength range $=300-360 \mathrm{~nm}$ ). Prior to supercritical methanolysis, the UV-cured resin sample was cryomilled into a fine powder by a freezer mill (Spex 6750) at liquid nitrogen temperature in order to improve the efficiency of methanolysis.

\section{Supercritical methanolysis procedure}

Figure 2 illustrates the apparatus for degradation used in this work. The autoclave was made of a stainless-steel (SUS 316) tube ( $1 / 2$ inch $\Phi, 13.5 \mathrm{~cm}$ long) with the inner volume of $10 \mathrm{ml}$, of which one end was sealed with a Swagelock cap. Twenty milligrams of the ground resin sample were put into an autoclave together with $7.5 \mathrm{ml}$ of methanol. Then, the autoclave was capped with a Swagelok union connected to a pressure gage to monitor the reaction pressure. The sealed autoclave was heated at around $300^{\circ} \mathrm{C}$ in a gas chromatograph oven. Under these conditions, methanol in the reaction tube was in a supercritical state over the critical point $(513 \mathrm{~K}$ and 8.1 $\mathrm{MPa}) .^{13}$ After heating for a prescribed period, the autoclave was cooled down in a water bath, the decomposed products were taken out of the autoclave and the inner wall of the tube was 


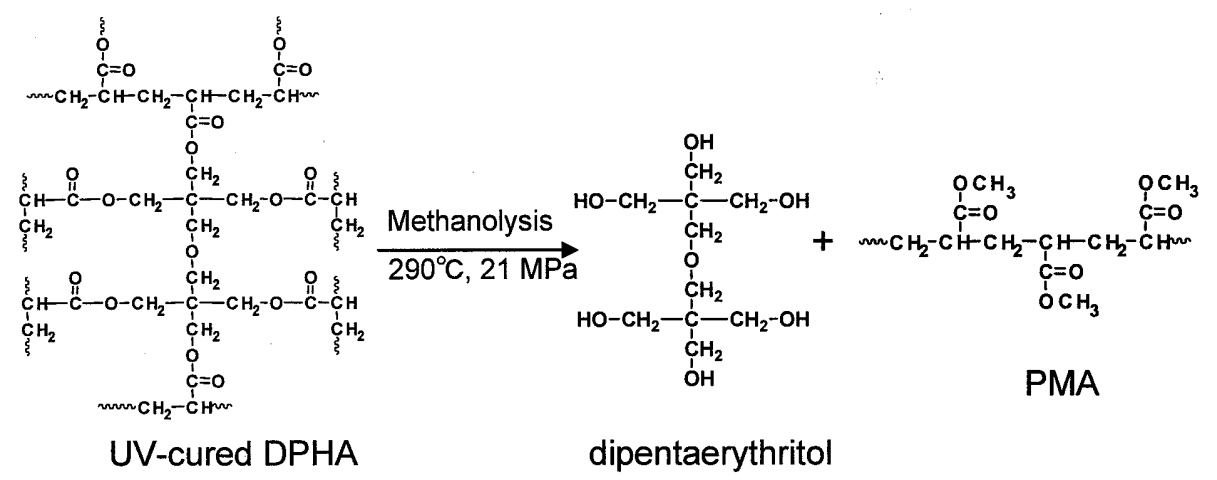

Fig. 3 Formation pathway of decomposed products of UV-cured DPHA in supercritical methanol.

washed out using tetrahydrofuran. The products in methanol were mixed with the rinsing solutions and dried in vасио, and then the obtained solid products were dissolved again with 0.15 $\mathrm{ml}$ of chloroform to be subjected to size exclusion chromatographic fractionation and/or MALDI-MS measurement.

\section{MALDI-MS conditions}

The combination of 1,8-dihydroxy-9[10H]-antracenone (dithranol; Sigma) and sodium iodide (Wako Pure Chemical Industries) was empirically selected as the optimum combination of matrix-cationization reagent and was used without further purification. First, $20 \mu \mathrm{l}$ of chloroform solution containing methanolysis products of the UV-cured resin was mixed with $20 \mu \mathrm{l}$ of tetrahydrofuran solution containing $0.1 \mathrm{M}$ dithranol and $0.01 \mathrm{M}$ sodium iodide. Then, $2 \mu \mathrm{l}$ of the sample/matrix mixed solution was spotted on a stainless-steel sample plate. MALDI-mass spectra were acquired using a timeof-flight mass spectrometer (AXIMA CFR Plus, Shimadzu/ Kratos) equipped with a pulsed nitrogen laser $(\lambda=337 \mathrm{~nm}, 3 \mathrm{~ns}$ pulse width) and a delayed extraction ion source. Ions generated by the laser desorption were introduced into the flight tube with an accelerating voltage of $20 \mathrm{kV}$. The delay time was set at $90 \mathrm{~ns}$. All mass spectra were acquired by averaging 100 individual laser shots under the default conditions of the spectrometer for mass calibration.

\section{Size exclusion chromatographic conditions}

Size exclusion chromatographic (SEC) measurements and fractionation were carried out using a liquid chromatograph system consisting of a pump (PU-2080 Plus; JASCO), a column oven (860-CO; JASCO) and a refractive index detector (RI2031 Plus; JASCO). Separation was performed by packed columns Shodex K-806M and K-803 (Showa Denko) connected in series. SEC instruments were operated at $40^{\circ} \mathrm{C}$ using chloroform as the mobile phase $(1 \mathrm{ml} / \mathrm{min})$. The values of $M_{\mathrm{n}}$ were calculated using a calibration curve generated with monodisperse polystyrene standards. To collect the fractions for MALDI-MS measurement this procedure was used: a $0.1 \mathrm{ml}$ chloroform solution of the methanolysis products was injected and $0.17 \mathrm{ml}$ of the eluent was taken every $10 \mathrm{~s}$ into a tapered polypropylene vial. After the solvent in the fraction was evaporated, the residue was mixed with $20 \mu \mathrm{l}$ of tetrahydrofuran solution containing $0.1 \mathrm{M}$ dithranol and $0.01 \mathrm{M}$ sodium iodide and the mixture was subjected to MALDI-MS measurement.

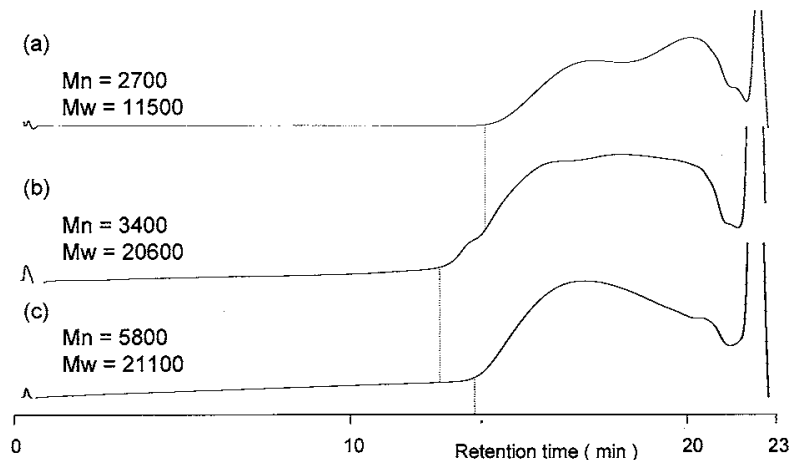

Fig. 4 SEC chromatograms of the products after supercritical methanolysis: (a) $300^{\circ} \mathrm{C}, 4 \mathrm{~h}$; (b) $290^{\circ} \mathrm{C}, 4 \mathrm{~h}$; (c) $290^{\circ} \mathrm{C}, 5 \mathrm{~h}$.

\section{Results and Discussion}

Optimization of supercritical methanolysis conditions

In the case of methanolysis of poly(ethylene terephthalate) (PET) using supercritical methanol, dimethyl terephtalate and ethylene glycol are mainly formed through trans-estrification. ${ }^{8}$ Provided that supercritical methanolysis of the UV-cured DPHA proceeds in the same manner as that of PET, selective cleavage and methylation should occur at ester linkages in the resin. Figure 3 illustrates the most probable formation pathway of the supercritical methanolysis products of the UV-cured DPHA. Methyl acrylate (MA) oligomers and/or polymers are to be formed through selective cleavage and methylation occurred at ester linkages in the UV-cured resins. Meanwhile, dipentaerithritol moiety should be converted to dipentaerithritol. Unless the pyrolytic cleavages at $\mathrm{C}-\mathrm{C}$ bonds in the sequence of acryloyl groups take place, the MA oligomers and/or polymers should reflect the chain length distributions of network junctions in the cured resin.

Figure 4 shows typical SEC chromatograms of the supercritical methanolysis products of the UV-cured DPHA decomposed under different conditions. In the chromatogram of the products decomposed at $300^{\circ} \mathrm{C}$ for $4 \mathrm{~h}$ (Fig. 4a), a continuous elution profile with two broad humps was observed. Although the first peak was thought to be chiefly comprised of poly(methyl acrylate) (PMA) formed through supercritical methanolysis, the second peak with longer retention time might be secondary degradation products formed through $\mathrm{C}-\mathrm{C}$ bond cleavage of PMA due to excess thermal energy. Meanwhile, in the middle chromatogram of the products formed through 


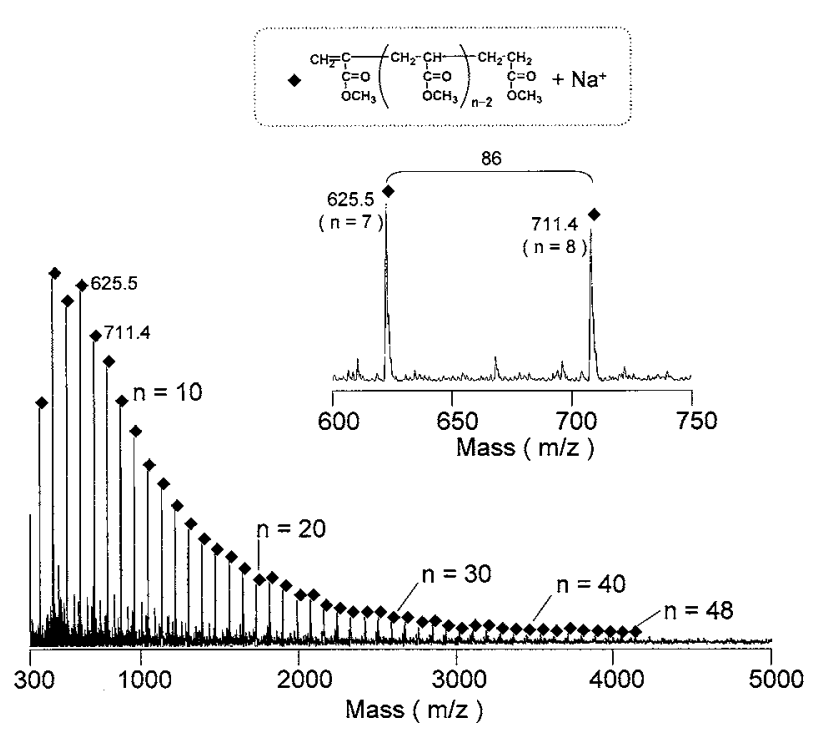

Fig. 5 MALDI-mass spectrum of supercritical methanolysis products of UV-cured DPHA decomposed at $290^{\circ} \mathrm{C}$ and $21 \mathrm{MPa}$ for $4 \mathrm{~h}$.

methanolysis at $290^{\circ} \mathrm{C}$ for $4 \mathrm{~h}$, a single broad peak eluting from ca. 13 min was mostly observed, indicating that undesirable pyrolytic cleavage was minimized. Moreover, it was confirmed that methyl methacrylate monomer subjected to these conditions did not polymerize, suggesting that polymerization of the decomposition products did not take place during supercritical methanolysis. However, a longer reaction time even at the same temperature caused a small decrease in the highest molecular weight of the products, probably due to the partial contribution of pyrolytic degradation observed in the bottom chromatogram eluting from about $13 \mathrm{~min}$, although the apparent average $M_{\mathrm{W}}$ values for (c) were slightly higher than those for (b). Consequently, supercritical methanolysis at $290^{\circ} \mathrm{C}$ for $4 \mathrm{~h}$ was selected as the optimum reaction condition for this work.

\section{Characterization of network structure in the UV-cured resin}

Figure 5 shows a MALDI-mass spectrum of the supercritical methanolysis products of the UV-cured resin obtained under the optimized conditions $\left(290^{\circ} \mathrm{C}, 21 \mathrm{MPa}\right.$ for $\left.4 \mathrm{~h}\right)$. In the mass spectrum, a series of peaks with the intervals of $\mathrm{m} / \mathrm{z}=86$ were observed in the mass range up to $c a$. 4000. The intervals of $\mathrm{m} / \mathrm{z}$ $=86$ are equal to a methyl acrylate monomer and the observed $\mathrm{m} / \mathrm{z}$ values of the peaks correspond to those of sodiumcationized PMA molecules having one vinylidene terminal as shown in Fig. 5. Accordingly, the network junctions in the UVcured resin were considered to be mostly decomposed into corresponding PMA in supercritical methanol under the optimized conditions.

However, the molar mass distribution of the PMA products in the mass spectrum in Fig. 5 was somewhat shorter than that estimated from the average molecular weight observed by SEC $\left(M_{\mathrm{n}}=3400\right.$ in Fig. 4(b)). This phenomenon was caused by a mass discrimination effect ${ }^{14-16}$ due to a broad distribution of PMA, reflecting a wide range of sequence lengths in the network junctions. In order to observe the whole range of PMA clipped out of the network junctions, therefore, the methanolysis products were first fractionated using SEC, and then the obtained fractions were subjected to MALDI-MS measurements.

Figure 6 shows the SEC chromatogram of methanolysis products of UV-cured DPHA together with the typical MALDI-

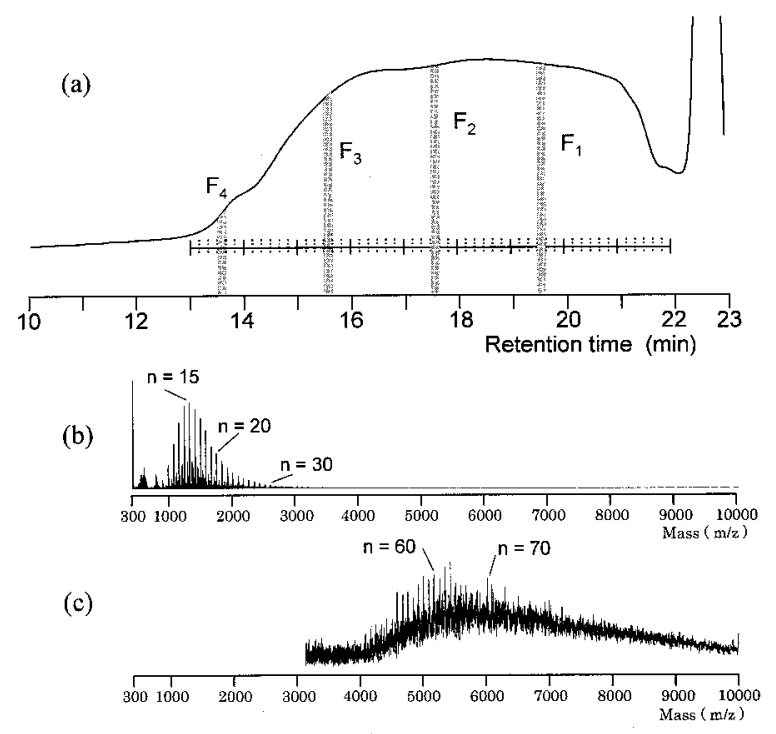

(d)

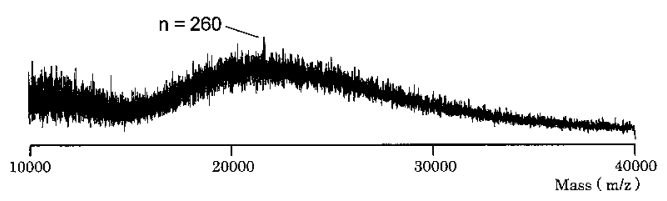

(e)

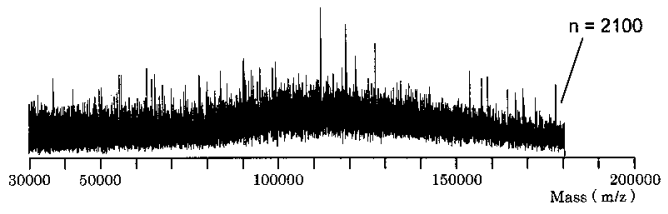

Fig. 6 SEC/MALDI-MS measurement of supercritical methanolysis products of UV-cured DPHA; (a) SEC curve of methanolysis products; mass spectra of (b) fraction $F_{1}(19.5 \mathrm{~min})$, (c) fraction $\mathrm{F}_{2}$ (17.5 min), (d) fraction $F_{3}(15.5 \mathrm{~min})$, and (e) fraction $F_{4}(13.5 \mathrm{~min})$.

mass spectra of the fractions taken at different retention times. In the mass spectrum of the fraction collected at $19.5 \mathrm{~min}$ (Fig. $6 \mathrm{~b}$ ), a series of peaks corresponding to the MA oligomers were mainly observed with the intervals of 86 mass units in the $\mathrm{m} / \mathrm{z}$ range between 1000 and 3000. Similarly, a number of peaks corresponding to PMA in the mass range up to $c a$. 6000 were clearly obtained in the mass spectrum of the fraction at $17.5 \mathrm{~min}$ (Fig. 6c). In addition, although the $S / N$ ratio was very low, a broad and non-resolved peak was observed in the mass spectra of the fractions having higher molecular weight (Figs. $6 \mathrm{~d}$ and e). The edge of the peak at $\mathrm{m} / \mathrm{z} \mathrm{ca} .180000$ in the mass spectrum of the fraction at $13.5 \mathrm{~min}$ (Fig. 6e) might be assigned to PMA with almost 2100 repeat units. Thus, these results demonstrated that the network junctions in the given UV-cured resin were composed of rather long polyacrylate sequences ranging up to about 2000 units in length.

In conclusion, MALDI-MS combined with supercritical methanolysis made it possible to characterize the cross-linking sequences of the UV-cured resin comprised of multi-functional acrylates. Collecting SEC fractions followed by MALDI-MS analysis could evaluate almost the whole range of molecularweight distribution of the polydispersed MA oligomers and polymers formed through supercritical methanolysis. This method would be useful to characterize rigidly cross-linked network structures in UV-cured acrylic ester resins widely used in practical applications. 


\section{Acknowledgements}

The authors are grateful to Dr. Hiroaki Sato [National Institute of Advanced Industrial Science and Technology (AIST)] for helpful discussions. This work was supported in part by the research foundation of Frontier Laboratory Inc., by a Grant-inAid for Scientific Research (B) (16350081) of Japan Society for the Promotion of Science, and by a Grant-in-Aid for Waste Management (K1734) of the Ministry of the Environment, Japan.

\section{References}

1. J. G. Kloosterboer, Adv. Polym. Sci., 1988, 84, 1.

2. J. L. Garnett, Radiat. Phys. Chem., 1995, 46, 925.

3. C. Decker, Prog. Polym. Sci., 1996, 21, 593.

4. A. B. Scraton, C. N. Bowman, and R. W. Peiffer, "Photopolymerization Fundamentals and Applications", ed. A. B. Scranton, C. N. Bowman, and R. W. Peiffer, 1996, Chap. 1, American Chemical Society, Washington, D.C., 1.

5. J. P. Fouassier, "Photoinitiation, Photopolymerization and Photocuring: Fundamentals and Applications", 1995, Chap. 6, Hanser Gardner Pub., OH, 246.
6. H. Matsubara, A. Yoshida, Y. Kondo, S. Tsuge, and H. Ohtani, Macromolecules, 2003, 36, 4750.

7. K. A. Burkoth and K. S. Anseth, Macromolecules, 1999 , 32, 1438 .

8. T. Sako, T. Sugeta, K. Ohtake, N. Nakazawa, M. Satoh, K. Namiki, and M. Tsugumi, J. Chem. Eng. Jpn., 1997, 30, 342 .

9. J. Ozaki, S. K. I. Djaja, and A. Oya, Ind. Eng. Chem. Res., 2000, 39, 245.

10. H. Sato, N. Ichieda, H. Tao, and H. Ohtani, Anal. Sci., 2004, 20, 1289.

11. H. F. Gruber, Prog. Polym. Sci., 1992, 17, 953.

12. H. Matsubara, A. Yoshida, H. Ohtani, and S. Tsuge, J. Anal. Appl. Pyrolysis, 2002, 64, 159.

13. R. C. Reid, J. M. Prausnitz, and B. E. Poling, in "The Properties of Gases and Liquid", 4th ed., 1987, McGrawHill Inc., New York, 670.

14. G. Montaudo, M. S. Montaudo, and F. Samperi, "Mass Spectrometry of Polymers", ed. G. Montaudo and R. P. Lattimer, 2002, Chap. 10, CRC Press, Boca Raton, FL, 419.

15. C. N. Peacock and C. N. McEwen, Anal. Chem., 2004, 76, 3417.

16. H. Pash and W. Schrepp, "MALDI-TOF Mass Spectrometry of Synthetic Polymers", 2003, Chap. 5, Springer-Verlag, Berlin, 107. 